\title{
La persuasion politique chez Pétrarque
}

Hélène Vonner

\section{OpenEdition}

\section{Journals}

Édition électronique

URL : http://journals.openedition.org/cei/250

DOI : $10.4000 /$ cei. 250

ISSN : 2260-779X

\section{Éditeur}

UGA Éditions/Université Grenoble Alpes

\section{Édition imprimée}

Date de publication : 15 avril 2005

Pagination : 41-61

ISBN : 978-2-84310-065-9

ISSN : 1770-9571

Référence électronique

Hélène Vonner, «La persuasion politique chez Pétrarque », Cahiers d'études italiennes [En ligne], 2 | 2005, mis en ligne le 15 octobre 2006, consulté le 27 mars 2021. URL : http:// journals.openedition.org/cei/250; DOI : https://doi.org/10.4000/cei.250 


\title{
LA PERSUASION POLITIQUE CHEZ PÉTRARQUE
}

\author{
Hélène Vonner \\ Université Nancy 2
}

\section{L'éloquence pétrarquienne et les exempla historiques}

Quanta vis esset eloquii, lepore simul et ratione conditi atque hinc verbis hinc sententiis affluentis, sepe re cognitum atque compertum est. Nunquam Milonis aut Herculis tam fortia brachia quin Ciceronis esset aut Demosthenis lingua potentior : illi quidem magno nisu molem forte aliquam, pondus ignobile ; hi vero animos movebant, rem nobilissimam penitusque mirabilem ac divinam.

Ainsi commence la Sen. II, 3, adressée à Francesco Bruni qui vient d'obtenir la charge de secrétaire apostolique ${ }^{1}$. Pétrarque s'attache à dénoncer les ennemis de l'écriture tout en exaltant la vigueur et le prestige de la parole et de la connaissance. Ne pas avoir peur des mots, savoir affronter les envieux et les contempteurs, rester humble ; voilà quelques-unes des règles énoncées par Pétrarque : il exhorte, enseigne, conseille, en puisant dans sa propre expérience d'homme de lettres. Il poursuit : "Optanda dictantibus lingua suavior, sed asperior metuenda ut semper placeas $»^{2}$.

Certes il convient de plaire, mais surtout d'être compris. En conséquence, comment écrire pour que le sens ne soit pas détourné, contesté, altéré ? Dans quelle langue, sous quelle forme, dans quel style? Il ne faut pas abuser des artifices littéraires ou d'expressions trop ampoulées, qui

1. Sen. II, 3, 1 : "Combien est puissante l'éloquence que grâce et raison viennent toutes deux agrémenter et qui regorge ici de mots, là d'idées, c'est ce qui a souvent été admis et démontré. Le bras de Milon ou celui d'Hercule ne fut jamais si fort que la langue d'un Cicéron ou d'un Démosthène ne le surpassât : ceux-là, en vérité, pouvaient peut-être, au prix d'un gros effort, soulever une masse, un poids aveugle ; mais ceux-ci savaient soulever l'esprit - l'objet le plus noble, et véritablement merveilleux et divin "; texte et traduction d'après Lettres de la vieillesse I-III - Rerum senilium libri I-III, éd. critique d'Elvira Nota, Paris, Les Belles Lettres, 2002, p. 156-157.

2. Sen. II, 3, 15: "Les écrivains doivent fuir l'âpreté et rechercher la douceur de la langue pour être continuellement plaisants" (ibid., p. 162-163). Dans cette optique, voir Fam. I, 9, 1. 
tendent souvent à masquer la véritable pensée de leur auteur ${ }^{3}$. Pétrarque connaît le pouvoir réel des mots sur l'auditoire : entre la persuasion et l'éducation d'autrui, il incarne le rôle de l'orateur antique, lequel enseignait la vertu et la gloire comme moteurs pour bien agir, se fondant sur les victoires des héros comme modèles à suivre. Il utilise cet art de convaincre qu'est l'éloquence pour critiquer la société dans laquelle il est obligé de vivre ${ }^{4}$, pour dépeindre une époque dissipée, pour tenter d'éveiller une nouvelle conscience civile grâce aux exemples et à la renommée des Anciens.

S'adressant cette fois à Tommaso da Messina dans la Fam. I, 9, à propos du studium eloquentie, Pétrarque écrit :

Quam multos, quibus nichil omnino loquentium exempla contulerant, etate nostra velut experrectos agnovimus et a sceleratissime vite cursu, ad summam repente modestiam alienis tantum vocibus fuisse conversos $!^{5}$

Comment expliquer mieux que cela l'efficacité de l'exemple ? Le culte de ces précieux repères permet de lutter contre la dépravation et la corruption; l'homme ne doit pas oublier les actions et les vertus passées, il doit chercher à les reproduire et à exploiter de la manière la plus appropriée ce trésor de sagesse et de grandeur. Si l'exemple fournit une preuve qui n'est qu'illustrative, qu'il montre plus qu'il ne démontre, il est toutefois la garantie d'une vérité, et doit s'inscrire dans un discours de persuasion pour pouvoir y trouver sa véritable fonction : justifier une assertion et permettre une démonstration. Selon la définition de Jacques Le Goff : «l'exemplum, venu de l'Antiquité gréco-romaine, est une anecdote de caractère historique présentée comme argument dans un discours de persuasion " ${ }^{6}$.

3. Cf. Fam. XII, 1, 4, à Charles IV : « [...] ideoque rem non nitor adornare coloribus, sciens ut coloratis fictisque mendacium, sic veritatem apertis ac simplicibus delectari " ( [...] et c'est pour cela que je ne m'efforce pas de parer d'ornements le style de ma lettre, sachant bien que le mensonge se complaît dans l'apparence et l'artifice, la vérité dans la franchise et la simplicité ") ; texte et traduction d'après Lettres familières Livres XII-XV-Rerum familiarium libri XII-XV, notices et notes d'Ugo Dotti, traduction d'André Longpré, Paris, Les Belles Lettres, 2004, p. 18-19.

4. Cf. De vita solitaria I, 9, 23 : « Hic sum, et mallem etate qualibet natus esse " (C'est ici que je me trouve, mais je préférerais être né à n'importe quelle autre époque ") ; texte et traduction d'après De vita solitaria - La vie solitaire, introduction, traduction et notes de Christophe Carraud, Grenoble, Millon, 1999, p. 158-159. Sur ce thème, voir essentiellement les Fam. II, 10, 3-4 ; III, 9, 4 ; VIII, 5, 13 ; XI, 9 , 1 ; XVI, 4, 4 ; XVIII, 1, 12-15 ; XX, 1 ; XX, 4, 7 ; XXIII, 19, 4 ; XXIV, 5, 2 ; XXIV, 8, 3, XXIV, 12, 27, répertoriées par Ugo Dotti, in Lettres familières Livres IV-VII-Rerum familiarium libri IV-VII, Paris, Les Belles Lettres, 2002, p. 489.

5. Fam. I, 9, 6, "Combien de nos contemporains n'avons-nous pas connus auxquels l'exemple n'avait été d'absolument aucune utilité, mais qui se sont réveillés pour ainsi dire et sont passés d'un coup d'une vie pleine d'infamie à une extrême modération à cause seulement de la parole d'autrui ! (Lettres familières Livres I-III - Rerum familiarium libri I-III, introduction et notes d'Ugo Dotti, traduction d'André Longpré, Paris, Les Belles Lettres, 2002, p. 100-101).

6. Jacques Le Goff, L’imaginaire médiéval. Essais, Paris, Gallimard, 1985, p. 9. 
L'écriture pétrarquienne va se révéler réceptive à ce procédé littéraire. Influencé par ses lectures, Pétrarque appelle fréquemment ses interlocuteurs à imiter un personnage exemplaire, à suivre le précepte d'un philosophe, d'un moraliste, d'un historien de l'Antiquité, ou encore à méditer certains dogmes des Saintes Écritures. Il souhaite surtout les guider dans leur quête du juste chemin ${ }^{7}$, les consoler au moment de la perte d'un proche $^{8}$, les aider lors d'un choix ou d'une décision difficile à prendre? ${ }^{9}$. Avec les exempla, le lecteur ne peut que se rendre compte de la véracité de l'allégation, et choisir d'y adhérer ou de la rejeter. Pétrarque, quant à lui, reste ainsi objectif et sûr de son propos. L'exemple forme une sorte de troisième interlocuteur, un tiers permettant d'éviter la stricte symétrie entre les deux correspondants; il représente à la fois un support littéraire ou historique, un soutien moral pour celui qui essaie de persuader, un pivot dans la discussion.

Grâce à la vaste érudition de l'auteur, les exempla envahissent rapidement l'ensemble des textes pétrarquiens, et un plaidoyer en faveur de la force des exemples apparaît dans la Fam. VI, 4. Dans cette lettre, intitulée Quid exempla valeant exemplis ostenditur ${ }^{10}$ et adressée au frère Giovanni Colonna $^{11}$, Pétrarque répond à une accusation portée contre lui concernant la profusion d'exempla dans ses écrits :

Possem, aiunt, paucioribus uti. Fateor, possem et sine exemplis agere ; nec istud infitior, quippe cum et tacere possem, et forte consultius. Sed in tot mundi malis, inter tam multa dedecora tacere difficile est. ${ }^{12}$

Il prouve leur intérêt incontestable dans une époque qui oublie peu à peu sa propre histoire; il remarque leurs effets stimulants non seulement sur sa propre personne mais surtout sur autrui, ou plus exactement sur ceux qui sauront en faire bon usage et en tirer un juste profit. Car il ne s'agit pas seulement de lire un exemplum, il faut le comprendre, le confronter au présent, y trouver un enseignement afin de corriger et redresser une situation personnelle ou collective. La dernière partie de cette lettre est consacrée à la narration de gestes d'éclat et de résolutions vertueuses, qui ont

7. Cf. Fam. III, 13 ; VI, 3 ; XXI, 9.

8. Cf. Fam. VII, 12 ; VII, 13 ; VIII, 1 ; XIII, 1.

9. Cf. Fam. XV, 1 ; XVII, 3 ; XXII, 5.

10. Fam. VI, 4 : « où il est montré par des exemples ce que valent les exemples » (éd. cit., p. 288-289).

11. Il ne s'agit pas ici du cardinal Giovanni Colonna, mais du frère dominicain en compagnie duquel Pétrarque a visité Rome lors de son premier voyage en 1337 ; voir Fam. VI, 2.

12. Fam. VI, 4, 1-2, «Je pourrais, dit-on, en user avec plus de parcimonie. J'en conviens. Je pourrais même m'en passer : cela aussi je ne le nie pas. Je pourrais encore me taire et cela serait peut-être plus raisonnable. Mais il est difficile de se taire quand les maux du monde sont si nombreux, ses turpitudes si nombreuses» (éd. cit., p. 288-291). 
éveillé le désir d'imitation chez certains, comme Jules César ou saint Augustin. Pétrarque démontre donc, par des exemples, l'importance, le rôle et la portée des exemples ${ }^{13}$.

Justifier un argument, l'illustrer, le clarifier, telles sont les fonctions de l'exemplum, qui doit également produire un phénomène d'identification, véritable motivation pour le progrès de l'homme.

\section{Le philosophe moral et son époque}

Pour Pétrarque, le souvenir des grands hommes évoqués dans les exempla ne peut que l'inciter à suivre lui-même leurs traces, regrettant de ne pas être à leurs côtés et désavouant l'époque où il est obligé de vivre :

Sicut enim horum graviter conspectus offendit, sic illorum recordatio magnificique actus et clara nomina incredibili me afficiunt atque inextimabili iocunditate, que si omnibus nota esset, multos in stuporem cogeret, quid ita cum mortuis esse potius quam cum viventibus delectarer. ${ }^{14}$

S'il trouve une forme de refuge dans ce dialogue avec les Anciens qui l'éloigne de l'inquiétude quotidienne ${ }^{15}$, Pétrarque va cependant essayer de confronter les remarquables événements du passé avec la réalité de son temps, pour aiguillonner peuple, seigneur, pape et empereur à sortir l'Italie de la décadence politique dans laquelle elle s'enlise.

Parce que le prestige du passé constitue un élément dynamique susceptible d'influencer l'ensemble de la société et de faire évoluer le cours des événements, Pétrarque va se lancer dans une mission identique à celle de l'orateur antique ${ }^{16}$ : exhorter, sous l'autorité des gloires passées, au redres-

13. Au sujet de ces auteurs considérés comme des modèles, Ernst Robert Curtius parle d' " autorités scientifiques ", de "source de savoir ", mais surtout de "trésor de sagesse ". Il nous apprend que Quintilien les nommait les sententiae et que "l'exemplum (paradeigma) est une expression de la rhétorique ancienne, qui date d'Aristote et signifie "histoire destinée à servir de pièce justificative" " (Ernst Robert CurTius, "Sententiae et exempla ", in La littérature européenne et le Moyen-âge latin, Paris, PUF, 1991 (première édition allemande : 1948), p. 115-120).

14. Fam. VI, 4, 5 : "De même en effet que la vue des uns me blesse profondément, de même le souvenir des autres, leurs actions admirables et leurs noms illustres me remplissent d'une joie incroyable et inestimable; si elle était connue de tous, beaucoup seraient stupéfaits que je prenne plaisir à être avec les morts plutôt qu'avec les vivants" (éd. cit., p. 290-291).

15. Selon Carlo Delcorno (Exemplum e letteratura. Tra Medioevo e Rinascimento, Bologne, Il Mulino, 1989, p. 231), " il modello eroico degli antichi trova la propria forza di persuasione, e la sua efficacia catartica proprio nella distanza che lo separa dalla mortificante realtà quotidiana " (" le modèle héroïque des Anciens trouve sa propre force de persuasion et son efficacité cathartique justement dans la distance qui le sépare de la mortifiante réalité quotidienne »).

16. Cf. Fam. X, 1, 12, à Charles IV : « [...] ut sic ego Carolum nostrum alloquar, sicut Iulium Cesarem Marcus Tullius alloquitur » (« [...] je vais parler à notre Charles comme Cicéron parlait à Jules 
sement de la péninsule. Le souvenir de l'histoire de Rome, de ses victoires, de l'essor de la cité, du respect des anciennes valeurs, doit fournir des règles pour évaluer le présent et comprendre le besoin réel d'une réforme politique, sociale et morale. À l'aide de modèles à suivre ou à éviter, il est désormais nécessaire d'éveiller les esprits, d'animer ce sentiment d'héritage à défendre et surtout à poursuivre. Il faut pousser les citoyens à s'engager et à se regrouper afin de renverser la triste situation actuelle.

Pétrarque oscille entre l'idéalisation du passé et l'aversion pour le présent. Les Lettres familières du dernier livre, qu'il adresse directement aux Anciens, s'élèvent au niveau de la diatribe contre le manque actuel de civilité et de civisme ; elles entrent dans un thème récurrent chez Pétrarque : la mutatio temporum. À ses yeux, l'homme est coupable des désordres sociaux et politiques, il est l'unique responsable de son propre malheur (" maxima pars malorum homo homini est ") ${ }^{17}$. Pétrarque s'attache à peindre les vices de cette société, regrettant la disparition des principes moraux, religieux et sociaux. Il règle ses comptes avec une société marquée par les troubles : les règles de vie sont transgressées, la corruption ne cesse de s'étendre, la soif de richesses est devenue la finalité de chacun.

Témoin des bouleversements de son siècle, Pétrarque ne peut s'empêcher de faire entendre ses condamnations et ses points de désaccord à l'intérieur des portraits élogieux qu'il dresse dans son De vita solitaria. Dans le deuxième livre, après l'évocation de la guerre de Cent ans, de la nonchalance du roi d'Espagne et l'impétuosité du roi du Portugal, après la dénonciation de la fuite de Charles IV et de la papauté hors d'Italie ${ }^{18}$, suit le jugement amer de Pétrarque :

Et hic quidem Europe status. Piget hinc longius proficisci, sed attingenda sunt vulnera, que, quanquam procul a capite et vitalibus membris, situ tamen et longa segnitie putruerunt. ${ }^{19}$

Au final, même l'ouvrage consacré à la vie solitaire est ponctué de remarques critiques sur une ère qui fuit la vertu et la vérité, pour laisser libre cours aux vices et aux impostures. Les hommes célébrés dans le traité -

César "; Lettres familières Livres VIII-XI - Rerum familiarium libri VIII-XI, notices et notes d'Ugo Dotti, traduction d'André Longpré, Paris, Les Belles Lettres, 2003, p. 240-241).

17. De remediis I, 88, 6: "L'homme est à l'homme l'essentiel de ses maux "; texte et traduction d'après De remediis utriusque fortune - Les Remèdes aux deux Fortunes, texte établi et traduit par Christophe Carraud, Grenoble, Millon, 2002, I, p. 382-383 ; cf. également De remediis I, 93, 12 : "Ratio. [...] animal ingratius homine nullum est» ("Raison. [...] c'est qu'il n'y a pas d'animal plus ingrat que l'homme» (éd. cit., p. 404-405); et De remediis I, 103, 18 ; I, 116, 15 ; II, 62, 2.

18. Cf. De vita solitaria, II, 9, 3-16 (éd. cit., p. 266-281).

19. De vita solitaria, II, 9, 7 : «Telle est la situation de l'Europe; je n'ai guère envie d'aller plus loin. Mais il faut tout de même toucher les blessures que leur emplacement et une longue incurie ont fait se putréfier ; il le faut, même si elles sont à distance de la tête et des membres vitaux " (ibid., p. 272-273). 
ermites, religieux, Pères de l'Église et philosophes - contrastent avec le peuple ignorant et les rois qui profitent de leur pouvoir. Les personnages du De vita solitaria deviennent par conséquent les exempla d'un monde qui semblerait presque devenu inconcevable aux temps de Pétrarque, tant les abus ont atteint toutes les classes sociales. Dans ce traité, Pétrarque constate les faits et les pose en parallèle avec les gloires passées. En ce qui concerne la thématique politique, l'auteur est plus contestataire que persuasif : il s'agit pour lui de dévoiler certains agissements et de les dénoncer. Dans le De remediis utriusque fortune, Pétrarque a, selon les mots de Ugo Dotti, " projeté sur la réalité trouble du présent et de la vie quotidienne, toujours incertaine, instable et capricieuse, l'expérience de sa longue méditation sur la culture stoïque et chrétienne ${ }^{20}$. La structure dialogique de l'œuvre et sa visée pédagogique conduisent le De remediis dans une logique de persuasion. Exposer les vanités du monde, retrouver la sérénité de l'esprit, apporter des remèdes par la voix de Ratio, tels sont les objectifs du traité. Par le biais d'un discours construit autour d'un raisonnement logique ainsi que sur de multiples exempla, il faut essentiellement exhorter le lecteur - ou les quatre interlocuteurs de Ratio - à regagner le chemin de la vertu et à contrôler les désirs. Si certaines pages rejoignent la polémique notamment lorsqu'il est question de problématiques relatives au pouvoir, à la richesse ou encore à la corruption, les thématiques du De remediis ne sont pas uniquement politiques, mais plus largement morales. Bien que l'œuvre soit dédiée à Azzo da Correggio, seigneur de Parme, et qu'elle soit dotée d'une fonction politico-didactique orientée vers la formation du nouveau prince, l'expression théorique reste l'empreinte majeure du traité. Outre les exemples, le De remediis se fonde sur des schémas logiques de démonstration, qui ne sont pas systématiques dans la persuasion politique. On trouve, en fait, des successions d'affirmations de la part de Gaudium, Spes, Dolor et Metus, auxquelles répond invariablement et méthodiquement Ratio. Prenons un exemple, le De rem. I, 116, De sperato principis adventu, et les assertions de Spes:

Principis adventum spero ; Spero principis adventum ; Spero principem venturum ; Principem venturum ego et respublica speramus ; Spero principem venturum ; Spero venturum principem ; Spero mox principem affuturum. ${ }^{21}$

20. Ugo Dotтi, Pétrarque, traduit par Jérôme Nicolas, Paris, Fayard, 1991, p. 245.

21. De remediis, I, 116 : "J'espère l'avènement d'un prince ; J'espère l'avènement d'un prince ; J'espère qu'un prince viendra ; J'espère, avec la République, l'avènement d'un prince ; J'espère qu'un prince viendra ; J'espère l'avènement d'un prince ; J'attends l'avènement prochain d'un prince » (éd. cit., p. 508-511; seules les répliques de Spes ont été reproduites ici). 
Raison développe ensuite une explication construite et concise, cherchant à troubler son interlocuteur, à le faire réagir, à créer un débat. Mais dans chaque dialogue, les répliques des quatre Passions de l'âme suivent une même ligne de répétitions, créant un leitmotiv auquel s'ajoutent lentement, au fil de l'échange, quelques variantes qui ne modifient cependant en rien leur position initiale. Elles sont intouchables, impossibles à détrôner, malgré les ruses argumentatives et les objections parfois lapidaires de Ratio ; celle-ci ne parvient pas à convaincre son/ses adversaire(s). La tentative de persuasion est bien présente dans le traité, mais elle se solde sans cesse par un échec, même si Raison a toujours le dernier mot dans le dialogue. Il manque en fait la présence de la volonté, que nous pourrions peut-être appeler Voluntas, créant un cinquième interlocuteur.

Dans le De vita solitaria et dans le De remediis, les discours de persuasion sont bien distincts. Le premier traité est essentiellement focalisé sur l'abondance des exempla, alors que le second est marqué par le rythme d'une écriture dialogique qui scande les arguments et la démonstration. Mais les intentions de l'auteur sont identiques : reconduire l'homme sur la voie du salut, lui prouver ses erreurs, le convaincre de rebrousser chemin à l'aide d'exemples issus de l'histoire.

Pour redresser les mœurs et la vie politique, il faut interpeller les puissants, ceux qui détiennent le pouvoir et qui sont susceptibles de changer le cours des événements. Dans cette optique, la correspondance devient une arme de persuasion politique efficace. Pétrarque n’hésite pas à condamner les inconduites et les regrettables entreprises des princes de son époque ${ }^{22}$, et c'est essentiellement dans les Rerum familiarium libri et les Sine nomine que l'on trouve ses plus vives critiques. Grâce à son couronnement capitolin du 8 avril 1341, Pétrarque est désormais un homme reconnu de tous ; il se rapproche ainsi des puissants et tente de les exhorter à reconstituer la grandeur de Rome et une politique italienne unitaire. Il faut réveiller une volonté d'action pour l'intérêt général depuis trop longtemps négligée, et qui permettra par la même occasion à celui qui opère d'obtenir une gloire personnelle. C'est sur ce point que semble s'établir la différence entre la persuasion rhétorique - présente dans le De remediis - qui agit sur le pathos de l'auditoire, et la persuasion politique qui a, en plus, cette possibilité

22. Cf. pour n'en citer qu'une seule, la Fam. VII, 15, 6, à Luchino Visconti : "Sed mutata sunt tempora ; reges terre bellum literis indixerunt ; aurum, credo, et gemmas atramentis inquinare metuunt, animum ignorantia cecum ac sordidum habere non metuunt " "Mais les temps ont changé. Les rois de la terre ont déclaré la guerre aux lettres ; ils craignent, je crois, de souiller d'encre leur or et leurs pierres précieuses, ils ne craignent pas d'avoir un esprit sordide et aveuglé par l'ignorance " ; Lettres familières Livres IV-VII, cit., p. 398-399). 
d'incliner la volonté d'autrui, car la volonté est précisément ce qui est mobilisé politiquement. Observons ainsi la persuasion politique chez Pétrarque, où les modèles issus de l'histoire antique et les chroniques contemporaines vont s'affronter dans des épîtres investies d'une dimension réformatrice et patriotique.

\section{Stratégies de la persuasion politique : les épîtres à Charles IV}

Le personnage de Scipion dans l'A frica, ainsi que les autres grandes figures présentées dans le De viris illustribus, deviennent chez Pétrarque l'exemple de vertu civique à suivre par ses contemporains. Avec l'Africa, Pétrarque est allé au-delà de la simple narration historique de l'héroïsme romain. Il a conjugué l'exaltation de la puissance militaire de la Rome antique à la célébration de la grandeur éternelle de la Rome chrétienne; l'histoire de la ville acquiert une dimension universelle. Engagé dans une mission didactique, le poète tente de ranimer l'image de la Capitale de la latinité. Il faut avant tout stimuler l'esprit de celui qui dirige la politique impériale. Dans sa critique de l'empire, Pétrarque confronte le dominant - l'Allemagne et le dominé - l'Italie - au milieu desquels se trouve Charles IV ${ }^{23}$. La dérobade de l'empereur aussitôt couronné, en avril $1355^{24}$, ne fait pas renoncer Pétrarque à son rêve de revoir un jour le siège du pouvoir impérial à Rome. Toutefois il hésite entre des espoirs pour ainsi dire démesurés, et la prise de conscience d'une certaine faiblesse de Charles IV.

Les Lettres familières d'exhortation envoyées au souverain se divisent en deux groupes. Les trois premières, antérieures au couronnement ${ }^{25}$, sont des lettres liminaires - Fam. X, 1 ; XII, 1 ; XVIII, 1 - et donc en position forte sur le plan rhétorique. Ces lettres sont imprégnées d'une volonté de mobilisation et d'une éloquence passionnée sur la gloire de Rome. Cette faconde devait amener l'empereur sur le sol italien, pour restaurer une souveraineté devenue inexistante à cause du fractionnement de l'Italie, et du pouvoir grandissant des seigneuries et républiques italiennes. Comme

23. Cf. De vita solitaria, II, 9, 6: "Germania nil aliud studet quam stipendiarios latrones in reipublice exitium armare [...]. Italia suis ipsa se viribus conficit " ( L'Allemagne ne songe qu’à armer des mercenaires pour la perte de l'État [...]. L'Italie met ses propres forces à s’affaiblir »; éd. cit., p. 272273).

24. Cf. De vita solitaria, II, 9, 5 : "Cesar hic noster rapto dyademate in Germaniam abiit patriis latebris et nomine contentus imperii " ("Notre empereur, après avoir ravi la couronne, est parti en Allemagne, pour s'y satisfaire du refuge de ses pères et du nom d'un empire "; éd. cit., p. 270-271).

25. La rédaction des Fam. X, 1 et XII, 1 se situe autour de 1350-1351, et celle de la Fam. XVIII, 1 entre 1352 et 1354 . 
dans l'Africa ${ }^{26}$, Rome prend la parole dans la Fam. X, 1, 15-25; elle se tourne vers Charles IV et retrace d'anciens triomphes, notamment l'entreprise de l'Africain. Puis elle lui demande de l'aider à se relever et lui annonce que grâce à une telle initiative, il entrera lui aussi dans la lignée des hommes illustres. Les espoirs politiques du poète et sa détermination à retrouver l'idéal du chef vertueux, exprimés dans l'Africa, renaissent dans la correspondance. Même si l'épître est ponctuée de reproches, Pétrarque mise sur la flatterie pour convaincre l'empereur : «te unum poscimus, tui siderei veltus intuitum postulamus $"{ }^{27}$.

L'appel à l'émotion est une des stratégies de persuasion. Entre la recherche de commisération à travers la prosopopée de Rome, et l'adulation frôlant presque la duplicité, Pétrarque joue avec le pouvoir des mots ; il ose : «non tam blanda quam querula oratione pulsaberis " ${ }^{28}$. Il importe d'attiser chez l'interlocuteur le zèle qui le conduira dans la péninsule. Pétrarque n'ignore pas l'exigence d'une image puissante pour réveiller Rome et l'Italie ${ }^{29}$; pour persuader Charles IV, il doit avant tout lui prouver qu'une telle opération ne peut qu'accroître son renom. Ici l'éloge participe à l'exhortation à l'action, dont la gloire personnelle représente la finalité suprême. Pétrarque incarne à présent le rôle du dispensateur de gloire, tel que l'incarnaient les poètes de l'Antiquité comme Horace ou Ovide $^{30}$.

La caractéristique de cette exhortatio apparaît dans la double prosopopée de Rome et d'Henri VII. La matrone romaine fait elle-même intervenir l'aïeul de Charles pour le convaincre de la nécessité d'une telle décision. La prosopopée n'apporte pas de preuve fondamentale à la démonstration, mais cette astuce littéraire permet d'éveiller l'émotion. Le choix de faire entrer en scène ces deux personnages n'est pas fortuit, car le

26. Cf. Africa VII, v. 506-660 : la prosopopée de Rome et Carthage s'adressant au maître de l'Olympe.

27. Fam. X, 1, 12: "c'est toi seul que nous demandons, nous voulons contempler ton divin visage " (Lettres familières Livres VIII-XI, cit., p. 240-241).

28. Fam. X, 1, 1: " ce n'est pas tant un discours flatteur qu'un discours plaintif qui te sera adressé " (ibid., p. 236-237).

29. Dans le De vita solitaria, II, 9, 14, il écrit à propos de Jules César : « [...] animi vim et acrimoniam illam miror ac necessariam temporibus nostris dico » (" [...] j'admire sa force d'âme, j'admire son énergie, et je les dis nécessaires aux temps qui sont les nôtres "; éd. cit., p. 279-281).

30. Cette thématique est évidente dans une autre lettre adressée à Charles IV, la Fam. XIX, 1, 2 : « [...] invenies, ne dubita, que tibi pollicitus sum, parata omnia : dyadema, imperium, immortalem gloriam apertumque celi aditum, et ad summam quicquid optare aut sperare datum homini "; cité d'après l'édition critique de Vittorio Rossi reproduite dans Francesco Petrarca, Opere, Florence, Sansoni, 1992, p. 999 («[...] tu trouveras, sois-en certain, tout préparé comme je te l'ai promis : la couronne, l'empire, une gloire immortelle et la voie ouverte vers le ciel, enfin tout ce qu' un homme peut désirer et espérer $")$. 
but est de toucher le lecteur, susciter tout d'abord en lui la commisération envers l'Italie, ensuite la confiance en cette opération et enfin l'ambition de la gloire. L'épître se conclut par une ultime incitation d'Henri VII envers Charles IV, le seul qui puisse désormais sauver l'Italie et de ce fait obtenir un vaste renom.

La seconde exhortation, la Fam. XII, 1, est dans la lignée de la précédente, mais insiste sur le fait que Charles doit se hâter d'assumer ses responsabilités politiques et de fixer son voyage en Italie. Cette fois ce n'est plus Rome qui se prosterne, mais l'auteur lui-même : «[...] oro precor obsecro, toto nunc animo genibus tuis affusus, quatenus occasionem res maximas atque optimas gerendi celitus oblatam incuntanter arripias ${ }^{31}$.

Pour analyser les procédés de Pétrarque dans ces deux lettres, nous pouvons nous appuyer sur les catégories développées par d'Aristote dans sa Rhétorique: l'éthos, le pathos et le logos. Nous retrouvons bien le caractère de l'orateur, sous-entendu Pétrarque (éthos), l'émotion du public et donc de l'interlocuteur Charles IV (pathos), le raisonnement illustré par les exemples (logos). Pétrarque adopte diverses tactiques pour persuader l'empereur, utilisant un discours emphatique et jouant sur des thématiques essentielles comme la fuite du temps et le pouvoir de la fortune.

Puis le ton change dans la Fam. XVIII, 1. Pétrarque parle plus ouvertement car les mois se sont écoulés et la situation est toujours inchangée. Il pensait pourtant avoir persuadé l'Empereur dans ses lettres antérieures ${ }^{32}$. Il analyse une après l'autre les trois excuses avancées dans la lettre Laureata tui que Charles IV envoya en réponse à la Fam. X, 1 : son renoncement à descendre dans la péninsule est dû au changement des époques, à la difficulté de conduire cette bête féroce qu'est l'Empire, à sa volonté de repousser l'utilisation des armes privilégiant la diplomatie. Dans cette troisième lettre d'exhortation, il n'est plus question de susciter l'émotion mais de solliciter la raison pour la survie de l'Italie. L'argumentation centrée sur l'ancienne gloire romaine prend désormais la place de la rhétorique flagorneuse des épîtres précédentes. C’est un véritable appel à la conscience historique et au poids de la tradition. À chaque excuse, Pétrarque apporte sa réponse et continue de vanter les mérites de la Rome antique : "Quis, oro te, divitem illam fecit, nisi civium suorum virtus ac modestie studium et iustitie cultus et rei bellice disciplina?" ${ }^{33}$.

31. Fam. XII, 1, 6: «[...] je te demande, je te prie et te conjure, humblement prosterné à tes pieds, de saisir sans hésiter l'occasion que t’offre le ciel d'accomplir de très grandes et d'excellentes actions " (éd. cit., p. 18-19).

32. Cf. Fam. XVIII, 1, 5.

33. Fam. XVIII, 1, 13 (Opere, cit., p. 959) : «Dis-moi, qui la rendit riche [Rome], si ce n'est pas la 
Examinons plus attentivement les stratégies de réfutation de Pétrarque. Il met en avant les incohérences dans le discours de Charles IV, en lui démontrant tout d'abord que les temps sont inchangés, virtus sola decre$v i t^{34}$. Puis en utilisant sans le nommer l'exemple de Cola di Rienzo, homme du peuple, il prouve qu'une action réformatrice est encore possible. Pour renverser la conclusion de son interlocuteur, Pétrarque adopte la contradiction, un procédé visant à montrer que l'adversaire n'est pas cohérent avec lui-même ${ }^{35}$. En réponse à la seconde excuse, il corrige l'empereur qui avait attribué la citation « quanta belua sit imperium » à Tibère et non à Auguste $^{36}$ : il s'agit cette fois d'une dissimilitude, puisque Pétrarque souligne l'amalgame erroné de Charles ${ }^{37}$. Tout en adhérant à cette idée, il faut renverser l'argument a contrario : puisqu'assurément l'Empire est une bête féroce, il ne pourra être dompté que par une main habile, celle de Charles IV. En revanche, une note ironique apparaît dans la dernière réponse : le souverain veut faire preuve de magnanimité à un moment où seule une opération militaire est susceptible de sauver la péninsule. "Nam quid amplius expectas ? Padus ut ad fontem redeat ? " ${ }^{38}$, écrit Pétrarque ; cet argument ad ignorantiam ${ }^{39}$ apporte une preuve contraire avec une tonalité acerbe afin de faire réagir l'adversaire. Après plusieurs renvois, bien évidemment attendus, aux exploits de l'Africain, de Jules César et de bien $\mathrm{d}^{\prime}$ autres ${ }^{40}$, l'exhortation reprend son caractère traditionnel, engageant l'interlocuteur à agir concrètement, toujours sous l'autorité des exempla. Mais dans la dernière partie de l'épître, Pétrarque contredit une ultime affirmation de Charles :

Addis autem - stupor auditu ! - nesciam tunc secessionis Italiam. Quando, obsecro, id fuit? ego enim in annalibus vix aut ullum tale tempus invenerim aut inveniri posse crediderim. $^{41}$

La réfutation entre dans les procédés de persuasion. En apportant la preuve que son correspondant se trouve dans l'erreur, l'auteur l'incite à se

\footnotetext{
vertu de ses citoyens, son habitude à la modération, son culte de la justice, la discipline de sa milice ?».

34. Cf. Fam. XVIII, 1, 14.

35. Cf. Bernard Dupriez, Gradus, Les procédés littéraires, Paris, 10/18, 1995 (1984), p. 391.

36. Cf. Fam. XVIII, 1, 21.

37. Cf. B. Dupriez, Gradus, cit., p. 391.

38. Fam. XVIII, 1, 28 (Opere, cit., p. 961) : «Et qu'attends-tu de plus ? Que le Pô retourne à sa source?".

39. Cf. B. Dupriez, Gradus, cit., p. 74.

40. Cf. Fam. XVIII, 1, 31-32.

41. Fam. XVIII, 1, 37 (Opere, cit., p. 963) : « Mais tu ajoutes - et je m’en étonne - qu'“alors l'Italie ne connaissait pas de rébellions". Et quand, je te prie, cela a-t-il pu arriver ? parce que moi, dans les annales, je ne trouve pas et je ne crois pas qu'on puisse trouver un tel moment ".
} 
remettre en question, à revenir sur ses positions, cherche à le convaincre et à stimuler sa volonté.

L'histoire romaine est devenue un symbole de perfection par sa supériorité militaire et culturelle. Aussi, dans l'absolu, Pétrarque voudrait voir Charles IV suivre les traces des Scipions et des Césars, imitant leur puissance combative et leur force de persuasion des troupes ${ }^{42}$. Mais la réalité sera tout autre : l'empereur pourra difficilement égaler les modèles pétrarquiens. Son manque d'intérêt pour la politique italienne traduit une faiblesse dans sa capacité de décision. Aussitôt après le couronnement, Pétrarque lui envoie la Fam. XIX, 12 pour lui reprocher son départ précipité, et lui faire remarquer que ses ancêtres et surtout son père n'auraient jamais agi de la sorte : "[...] a nullo unquam principum tantam spem, tam floridam tam maturam tamque honestam, sponte desertam $»{ }^{43}$.

Le second groupe d'increpationes est rassemblé dans le livre XXIII : Fam. 2, 15, 21. Ces lettres expriment désormais impatience, critiques et incompréhension après cette dérobade injustifiée et impardonnable pour Pétrarque. La Fam. XXIII, 2 est divisée en trois parties : d'abord Pétrarque remercie Charles de la bienveillance qu'il lui porte, lui démontre grâce aux exempla l'inexorable fuite du temps et que la vie d'un prince est plus brève que toutes les autres. Il tente de le convaincre de s'investir maintenant, de ne pas se perdre dans de longues réflexions qui le retardent, et de ne pas remettre à demain ce qu'il doit faire aujourd'hui. Ensuite surgissent les reproches relatifs à sa fuite dès qu'il reçut la couronne impériale. Pétrarque exprime son opinion avec une simplicité brutale : «Nichil omnino evenire potest, quod in te oblivionem excuset imperii $"{ }^{44}$.

Alexandre, Jules César, la famille des Scipions, ce sont toujours les mêmes figures qui reviennent et qui animent l'écriture pétrarquienne. Les sentiments antagonistes d'espoir et de déception ne font que refléter l'opiniâtreté de l'auteur, qui voudrait voir renaître la suprématie romaine avec Charles IV. Enfin, la dernière partie de l'épître est une nouvelle exhortatio

42. Cf. Fam. XIX, 3, 15, lorsque Pétrarque offre à Charles IV des pièces d'or et d'argent à l'effigie d'anciens empereurs et lui dit (Opere, cit., p. 1004) : " "Et ecce" inquam, "Cesar, quibus successisti ; ecce quos imitari studeas et mirari, ad quorum formulam atque imaginem te componas" " ("Voici”, dis-je, "ô César, ceux auxquels tu as succédé ; voici ceux que tu dois essayer d'imiter, en te conformant à leur pensée et à leur personne” »). Dans la Fam. XXIII, 1, indirectement adressée à Charles IV, Pétrarque apostrophe les anciens héros qui ont contribué à la gloire de Rome : Brutus, Camille, Scipion, Paul Emile, Marius, Pompée, Jules César, Auguste, Vespasien, Titus, Trajan et Théodose ; il regrette leur disparition et souhaite que Charles IV poursuive leurs œuvres.

43. Fam. XIX, 12, 4 (Opere, cit., p. 1024) : «[...] aucun prince n'abandonna jamais spontanément un espoir aussi grand, aussi florissant, aussi mûr, aussi honnête ».

44. Fam. XXIII, 2, 29 (Opere, cit., p. 1195) : "Rien ne pourra jamais arriver qui puisse t'excuser d'avoir oublié l'Empire ". 
scandée par les impératifs " expergiscere ", " accelera ", "surge ", " confrica ", "circumspice ", " aude ", car Pétrarque ne désespère pas et croit toujours en son idéal. Il joue fréquemment sur ces arguments a fortiori ${ }^{45}$ pour atteindre l'ego de Charles (puisque tu es né pour l'Empire, montrenous ta grandeur d'empereur, ta force et ta fermeté), et fait également appel à des témoins fictifs ${ }^{46}$, d'une part les Italiens contemporains de Pétrarque, d'autre part la postérité (quelle image garderont de toi les générations futures ?). Tous les stratagèmes sont bons pour incliner la volonté du souverain.

La Fam. XXIII, 15 prend une tonalité fataliste. Nous sommes en 1363 ; deux ans se sont déjà écoulés depuis la précédente exhortation. L'écriture entremêle l'éloge et la condamnation envers celui qui a fait naître de grands espoirs pour mieux les étouffer dans sa fuite. Créant un parallèle avec la figure de l'amant désenchanté, Pétrarque se rend à l'évidence et met en scène son acte de persuasion par l'écriture ${ }^{47}$ : il a maintenant épuisé tous ses arguments pour convaincre, aussi :

Et michi et tuis omnibus hoc unum superest, ut te in animo habeamus numinis instar sed aversi et nostras miserias non curantis. ${ }^{48}$

Il adresse néanmoins une dernière lettre d'exhortation à l'Empereur, la Fam. XXIII, 21. Plus conciliante que les précédentes, elle est l'occasion pour Pétrarque d'insister sur l'importance d'une gloire acquise par de nobles desseins, une thématique qui rappelle la Fam. X, 1 :

$[\ldots]$ is cum venerit, e cuntis opibus tuis atque imperii nil tibi supererit, nisi quantum ad eternam anime vitam et nominis immortalem gloriam bene vivendo quesieris. ${ }^{49}$

Comme dans l'Antiquité, il est nécessaire de transmettre à la postérité des œuvres justes et louables pour la survie de son renom ${ }^{50}$. Pétrarque essaie à nouveau d'éveiller l'émotion chez son interlocuteur en utilisant la flatterie. Mais est-il vraiment sincère en consentant une future immortalité à Charles IV, obtenue grâce à la " grandeur " de ses entreprises ? Le doute s'installe, car comme le remarque Ugo Dotti, Charles IV est exclu du De viris illustribus, malgré l'insistance de l'intéressé, car il n'a pas mérité d'y

45. Cf. B. Dupriez, Gradus, cit., p. 72.

46. Cf. ibid., p. 74

47. Cf. Fam. XXIII, 15, 5.

48. Fam. XXIII, 15, 5 (Opere, cit., p. 1225) : "Pour tous les tiens et pour moi, il ne nous reste plus qu'à te garder dans notre esprit comme notre dieu, mais un dieu distant et insoucieux de nos misères ".

49. Fam. XXIII, 21, 9 (ibid., p. 1237-1238) : « [...] lorsqu' elle viendra [la mort], de toutes tes richesses et de l'empire, il ne te restera rien, sauf ce que tu auras acquis en vivant honorablement pour la vie éternelle et pour la gloire immortelle de ton nom ».

50. Cf. Africa III, v. 481-486. 
figurer $^{51}$. Le De viris sera dédié à François de Carrare ; une préface particulière lui sera même consacrée ${ }^{52}$, et il trouvera ainsi sa place aux côtés des hommes illustres. Et le critique italien poursuit en signalant que l'intellectuel « si propone come l'unico autorizzato a dare, con il proprio indiscusso giudizio, fama e immortalità agli uomini " ${ }^{53}$.

Grâce à son éloquence, Pétrarque s’investit de cette fonction de médiateur entre la gloire et celui qui la mérite. Il participe à l'entretien de la renommée et cherche à dispenser un enseignement où seul le passé sera susceptible de transmettre des valeurs sûres. Comme pour les troupes de l'Africain, la gloria représente un facteur d'union, qui doit permettre de sortir Rome et la péninsule de cette torpeur civique. Loin d'être un simple artifice littéraire, la peinture du monde romain suggère une homogénéité spirituelle, et le modèle des gloires passées acquiert un fondement politique et une conception moralement juste et primordiale.

Influencé par les modèles de l'Antiquité, fier de l'ancienne grandeur romaine et enthousiasmé par la possible résurrection du passé, Pétrarque propose son enseignement historique et moral à celui qui détient l'autorité, qui pourra s'identifier aux héros romains, qui devra stimuler les troupes pour réformer l'État. Le nouvel humaniste n'a pas de théorie politique clairement définie à défendre, il vise surtout le retour d'un prestige, et se trouve parfois divisé entre la réalité et l'idéal. Comme le souligne Henri Lamarque, après la mort du roi Robert Pétrarque a cherché en la personne de Charles IV « son idéal du prince ou du grand meneur d'hommes, [...] l'homme politique auquel il pouvait accrocher ses rêves " ${ }^{54}$.

51. Cf. Ugo Dotri, Petrarca civile, Rome, Donzelli, 2001, p. 212. Cf. Fam. XIX, 3, 13, récit d'une rencontre entre Pétrarque et Charles IV : "Quod autem ad te, Cesar, ita demum hoc te munere et eius libri titulo dignum scito, si non fulgore nominis tantum aut inani dyademate, sed rebus gestis et virtute animi illustribus te te viris ascripseris et sic vixeris ut cum veteres legeris, tu legaris a posteris " (Opere, cit., p. 1004 : «En ce qui te concerne, ô César, sache que tu seras vraiment digne du don et de la dédicace de ce livre [le De viris], non seulement par la splendeur de ton nom et par la vanité de ta couronne, mais aussi lorsque, par tes actions et par ta vertu, tu te rangeras au nombre des grands hommes et que tu vivras en sorte que, comme toi tu lis la vie des anciens, de même la postérité lise la tienne»).

52. Cf. Francesco Petrarca, De viris illustribus, a cura di Guido Martellotti, Florence, Sansoni, 1964, I, p. XIII-XIV.

53. U. Dotтi, Petrarca civile, cit., p. 212 (l'intellectuel « se propose comme étant la seule personne autorisée à accorder, grâce à son jugement incontestable, la renommée et l'immortalité aux hommes "). Cf. Fam. XIX, 3, 24, la remarque d'un soldat toscan à Charles IV à propos de Pétrarque : "Ecce” inquit, "imperator, de quo tibi sepe dixeram, qui siquid laude dignum gesseris, nomen tuum tacitum non sinet; alioquin et loqui didicit et tacere” " (Opere, cit., p. 1006 : "Voici”, dit-il, “ô César, celui dont je t'ai souvent dit que, si tu accomplis des choses dignes de louanges, il ne taira pas ton nom ; dans le cas contraire, il saura parler et se taire"»).

54. Francesco Petrarca, Africa - L’Afrique, traduit par Rebecca Lenoir, Grenoble, Millon, 2002, préface, p. 17. 
Pétrarque a-t-il vraiment réussi à convaincre Charles IV de venir en Italie, ou est-ce la succession des événements et les relations favorables avec Innocent VI qui l'en ont persuadé ? La poursuite - devenue presque obsédante - de son idéal, plonge parfois Pétrarque dans une grandiloquence passionnée, où l'écriture se dote d'une dimension épique jusque dans la correspondance. Mais Pétrarque s'est surtout réfugié dans le mythe pour démontrer la volonté d'un homme qui désire s'affirmer sur la scène politique. Il prouve qu'il n'est pas seulement un poète, un moraliste et un philosophe, mais qu'il sait analyser le monde qui l'entoure, tirer les leçons de son érudition, pour devenir non pas un homme politique à part entière, mais un intermédiaire politique, notamment grâce à l'influence de sa plume, de plus en plus prisée par le public. C'est un homme public, et par cette position il s'affranchit des partis, ne dépendant finalement de personne sauf de lui-même.

\section{Les épîtres à Cola di Rienzo}

Après la fuite de la papauté en Avignon, Rome orpheline est en proie aux guerres civiles $^{55}$. La noblesse romaine s'affronte pour le pouvoir et deux clans s'opposent : les Orsini et les Colonna. Laction devient plus que nécessaire, c'est pourquoi l'entreprise de Cola di Rienzo ${ }^{56}$, commencée en 1347, provoque immédiatement l'adhésion enthousiaste de Pétrarque ${ }^{57}$. Il se déclare en faveur du tribun afin qu'il proclame son décret du $1^{\mathrm{er}}$ août 1347, grâce auquel les communes italiennes redevenaient libres, tous les Italiens étaient citoyens romains, et Rome retrouvait sa position de capitale de l'Empire et de la Chrétienté, incarnant à nouveau son rôle de caput orbis. Les desseins de Cola ne pouvaient qu'enflammer l'âme de Pétrarque, qui voyait déjà se concrétiser son rêve d'un retour à la démocratie antique et la fin de la guerre entre les factions romaines. Il avait besoin de croire en celui qui incarnait enfin le renouveau, et il lui adresse en septembre 1347 les Sine nomine II et III ${ }^{58}$. À propos de ces deux lettres, Ugo Dotti écrit :

55. Cf. De remediis II, 69, 2 : «Ratio. Romam nullus in totum diruit ; sed senectus attrivit, civilibus adiuta discordiis " ("Raison. Personne, il est vrai, n’est parvenu à détruire Rome ; mais la vieillesse l'a usée, avec le triste concours des guerres civiles "; éd. cit., p. 836-837).

56. Pour une étude approfondie sur Cola di Rienzo, voir Paul PIur, Cola di Rienzo, traduzione di Jeanne Chabot Robir, Milan, Treves, 1934.

57. Sur les relations entre les deux hommes, voir Francesco Ercole, "Petrarca e Cola di Rienzo ", in Annali della cattedra petrarchesca, VI, 1935-1936, p. 85-103 ; et Josef MACEK, "Pétrarque et Cola di Rienzo ", in Historica, XI, 1965, p. 5-51.

58. Pétrarque enverra à Cola également les Var. 48 et 38 juste avant ce fameux mois d'août 1347 , 
"Mi sembra indubbio che esse siano state dettate sotto l'urgere di uno sdegno più politicamente appassionato e dietro la spinta di un evento storico più immediato ed esaltante $" 59$.

Dans la Sine nomine II, Pétrarque réagit tout d'abord à l'agression subie par l'un des messagers de Cola aux portes d'Avignon. Il exhorte Cola à poursuivre son entreprise malgré l'hostilité de la curie avignonnaise, et conclut non sans une certaine détermination : "Vale et perfice quod cepisti ! ${ }^{60}$. L'enthousiasme et la motivation de Pétrarque se révèlent pleinement. Les relations qu'il entretenait avec la curie pontificale et les Colonna ne mettent pas un frein à la fermeté de ses convictions à l'égard du tribun et son audacieuse entreprise - bien au contraire. La stratégie de persuasion envers Cola fait encore appel au pathos. Il n'est plus question d'enjôler l'interlocuteur, mais plutôt de l'informer de la foi que l'on a dans son entreprise. Pétrarque partage les idées du tribun et souhaite que son projet aboutisse ; il veut lui apporter son soutien, un soutien moral certes, mais aussi politique. S'engager auprès de Cola ne lui suggère aucune crainte vis-à-vis de ses protecteurs. En 1347 s'amorce la rupture entre Pétrarque et les Colonna ${ }^{61}$; l'homme de lettres cherche désormais son indépendance pour pouvoir exprimer clairement ses idées. Faire abstraction des intérêts particuliers et attiser une énergie collective italienne, telle est l'idéologie qui émane de la figure de Cola aux yeux de Pétrarque. Cola incarne, au même titre que l'empereur, un idéal ; l'idéal d'un homme qui s'emploie au bien de tous les Italiens, qui est doué de ce courage, digne des anciens romains, d'affronter les puissants et d'affirmer ses idées sur la scène politique.

Mais à la différence des Lettres familières adressées à Charles IV, ici, plus qu'une tentative de persuasion du tribun, Pétrarque semble s'être persuadé lui-même de la concrétisation du retour à l'idéal romain. Il extériorise son indignation face à la décadence avignonnaise ${ }^{62}$, et donne parfois l'impres-

puis les Var. 42 et 40 pendant le mois d'août. Il y célèbre la liberté reconquise par le peuple romain et informe Cola de la naissance d'une certaine méfiance de la part de la papauté envers lui ; voir Francesco Petrarca, Sine nomine. Lettere polemiche e politiche, a cura di Ugo Dotti, Rome-Bari, Laterza, 1974, p. XI-XII.

59. Ibid., p. XI : «Il me semble certain que celles-ci aient été dictées par l'urgence d'une indignation passionnément politique et sous l'impulsion d'un événement historique plus immédiat et exaltant ".

60. Sine nomine II, 5 (éd. cit., p. 44-45) : «Portez-vous bien et finissez ce que vous avez commencé ! ». Nous empruntons la traduction de Rebecca Lenoir (F. PÉTrarque, Sans titre - Liber sine nomine, Grenoble, J. Millon, 2003).

61. Cf. la huitième églogue du Bucolicum carmen intitulée Divortium.

62. Sine nomine II, 5 (éd. cit., p. 42-43) : "Hec levandi animi gratia dixisse volui » ( Je vous ai écrit tout cela parce que je voulais décharger mon âme "). 
sion d'écrire pour lui-même : «[...] nec impulsore nec lenitore indiges, et calcar et frenum in potestate habes $"{ }^{63}$.

Dans l'épître suivante, la Sine nomine III, Pétrarque informe Cola de la décision de quelques cardinaux, réunis à Avignon, de ne pas unifier Rome et l'Italie, et donc de ne pas suivre les idées annoncées dans le décret du tribun :

Et quamvis ipsa rerum dubitatio satis puerilis et inepta sit, tamen disputandi studio utcumque poterat excusari, nisi multis argumentis ultro citroque iactatis is, qui omnium sapientissimus habebatur, cum plausu et favore omnium : " Nullatenus expedire " venenata diffinitione firmasset. ${ }^{64}$

Il dénonce ouvertement l'injustice d'un tel verdict et demande à Cola de propager, tel un orateur antique, la nouvelle dans Rome et dans toute la péninsule. Toujours sous l'égide de cette fides, il lui confie une mission, il a foi en lui et lui fait confiance.

Mais la papauté, qui avait au départ une opinion favorable de Cola, se fait de plus en plus réticente. De plus, le morcellement grandissant de l'Italie, les ambitions démesurées du tribun et ses erreurs de stratégie politique et diplomatiques vont lui être fatales. Dans la Fam. VII, 7 du mois de novembre 1347, la seule du recueil qui soit adressée à Cola, Pétrarque critique son changement d'attitude :

[...] non te populum, ut solebas, sed partem populi pessimam amare, illi obsequi, illam colere, illam admirari. [...] ubi nunc ille tuus salutaris genius, ubi, ut usitatius loquar, ille bonorum operum consultor spiritus, cum quo assidue colloqui putabaris ? ${ }^{\circ}$

Pourtant Pétrarque continue à espérer en la réussite du projet, en encourageant le tribun à réfléchir, à revenir sur sa position et surtout, à ne pas oublier le déclin de sa brillante renommée s’il venait à faillir à ses engagements ${ }^{66}$. Hélas, contraint d'abdiquer fin 1347, Cola fuit Rome et se retrouvera prisonnier d'abord à Prague, puis à Avignon pendant l'été 1352 jusqu'au mois de septembre 1353. Si le tribun déchu était officiellement

63. Sine nomine II, 4 (ibid., p. 40-41) : « [...] vous n'avez besoin ni d'être exhorté ni d'être retenu, car vous possédez la capacité de vous stimuler et de vous réfréner vous-même ".

64. Sine nomine III, 2 (ibid., p. 46-49) : "Bien que la question soit en elle-même assez stupide et puérile, l'amour de la discussion aurait pu suffire à la justifier, si celui qui faisait office du plus sage, après que l'on eut débattu du pour et du contre avec force arguties, n'y avait mis un point final et venimeux, dans l'approbation et l'admiration générale, en affirmant que "de toute façon, ce serait une mauvaise chose!"”.

65. Fam. VII, 7, 7-8: «[...] tu n'aimes plus le peuple, comme c'était ton habitude, mais la partie la pire du peuple; tu lui obéis, tu l'entoures de tes soins, tu l'admires. [...] Où est maintenant ton génie salutaire, où est, pour parler plus communément, ton esprit qui te conseillait les bonnes œuvres et avec lequel, pensait-on, tu conversais régulièrement ?" (Lettres familières Livres IV-VII, cit., p. 360-361).

66. On retrouve ici une tentative de rhétorique flatteuse pour persuader le tribun. 
accusé d'avoir investi le Capitole et d'avoir voulu restituer à Rome le gouvernement de l'Empire, Pétrarque quant à lui n'avait qu'un seul reproche à lui faire : la défaite de son action et donc l'évanouissement d'un espoir ${ }^{67}$. Désappointé, il critique la papauté pour avoir emprisonné le tribun, mais désapprouve l'indulgence de ce dernier envers la noblesse romaine, une erreur qui fut à l'origine de sa perte. Au-delà d'un simple désir de retour de la tradition antique, c'est un sentiment de lutte contre le pouvoir temporel détenu par la papauté qui s'élève derrière l'action de Cola di Rienzo, pour la formation d'une société laïque et d'une nouvelle conscience civile.

Bien qu'il n'ait pas pu vivre pleinement cette révolution à cause de la barrière des Alpes, l'homme de lettres y avait cru avec fermeté, sans doute aveuglé par une trop grande exaltation. Ainsi, dans les Fam. XI, 16 et 17, il se tourne vers les quatre cardinaux chargés en 1351 par Clément VI de la réforme du gouvernement de Rome. Selon le poète, il faut lutter contre la noblesse romaine, réformer le Sénat et laisser la place aux citoyens romains. Bien que Pétrarque ait été très proche des Colonna, il déclare avec conviction :

[...] alteram non odisse, alteram vero, quod commemorare superfluit, non amare solum, sed familiari quodam semper obsequio coluisse, nullamque michi toto orbe principum familiam cariorem ; carior tamen michi respublica, carior Roma, carior Italia, carior bonorum quies atque securitas. ${ }^{68}$

Ce sentiment profond de romanité et la volonté de sauver la République s'accentuent dans les Sine nomine VII et IX de novembre 1351, toujours à propos de cette réforme : à nouveau Pétrarque s'insurge contre l'oppression involontairement subie mais pourtant consciemment acceptée par le peuple romain.

Mais l'aventure de Cola di Rienzo ne fut pas totalement vaine pour Pétrarque, puisque c'est à partir de cet épisode que naît l'initiative de rompre les liens avec la curie et de quitter la Provence pour aller s'installer en Italie. Il passe du service de Giovanni Colonna à celui qui incarne l'idéal

67. Sur le rôle providentiel de Cola selon Pétrarque, voir Johannes BARTuschat, « La révolution manquée de Cola di Rienzo à travers les témoignages contemporains ", in Soulèvements et ruptures : l'Italie en quête de sa révolution. Échos littéraires et artistiques (PRISMI, II), Nancy, Université de Nancy, 1998 , p. 11-31, où est mise en évidence une opposition entre la vision, sûrement trop idéale, de Pétrarque face à la figure du tribun, et celle de l'Anonyme romain, contemporain des deux hommes, qui, dans sa Cronica s'attache à son rôle d'historiographe et relate en détail la dure réalité des faits.

68. Fam. XI, 16, $8:$ :[...] je ne hais pas l'une des deux familles [les Orsini] qui sont à l'origine de cette querelle ; quant à l'autre [les Colonna] - il est bien superflu de le rappeler -, non seulement je l'aime mais j'ai toujours manifesté à son égard respect et déférence, et il n'y a aucune autre famille princière dans tout l'univers qui me soit plus chère. Plus chère toutefois m'est la république, plus chère Rome, plus chère l'Italie, plus chers le repos et la sécurité des gens de bien » (éd. cit., p. 404-405). 
du prince-mécène, Giovanni Visconti : du cardinal au seigneur de Milan, du spirituel au temporel; Pétrarque s'affranchit et ne craint pas d'imposer ses idées ${ }^{69}$. C'est sans scrupule qu'il tourne le dos à Avignon pour devenir, dans cette nouvelle société en formation, porte-parole du prince. Ugo Dotti évoque même une "missione "illuministica" che lo scrittore avverte come dato fondamentale e come compito precipuo dell'intellettuale ormai uscito dalle strette della cultura medievale ${ }^{70}$.

\section{La persuasion politique et la nouvelle image de l'intellectuel}

\section{Dans la Sine nomine II, Pétrarque écrit :}

De reliqua enim Italia, cui dubium est quin quantum potuit possit, nec consilium nec vires nec opes nec animos defore, sed consensum ? ${ }^{7}$

Décalage de l'intellectuel par rapport à son époque ? Il n'en est rien. Pétrarque sait observer cette société du Trecento, et tente de l'interpréter en s'armant de sa vaste érudition. Mais à travers la persuasion d'autrui, il démontre ses talents d'érudit et d'intellectuel médiateur. Entre l'orateur et le prédicateur, il trouve sa place dans cette société qui se transforme.

Pétrarque incarne la figure du nouvel intellectuel au service de ceux qui détiennent l'autorité. Son écriture se veut didactique, et elle expose les idées de celui qui est devenu un nouveau maître de morale. Dans les épîtres que nous venons d'analyser, Pétrarque s'efforce davantage de persuader que de plaire. Ses exempla ne s'inscrivent pas dans une dimension ornementale, mais leur rôle est de convaincre le destinataire du bien-fondé de ses propos, et de la nécessité de les concrétiser par des actes. Il ne cherche pas à entrer en guerre contre les puissants, puisqu'il sait qu'eux seuls ont la possibilité de renverser le cours des événements, s’ils en ont la volonté et le désir. Ce qui le distingue de Dante, dont certaines lettres, notamment l'épître VI aux Florentins ${ }^{72}$, rappellent les Sine nomine, c'est une concep-

69. On comprend maintenant l'audace de Pétrarque dans la Fam. XI, 16, critiquant les Colonna dans leur lutte contre les Orsini. En 1351, date de rédaction de l'épître, une volonté d'indépendance commence à s'affirmer dans l'esprit de l'homme de lettres.

70. Sine nomine. Lettere polemiche e politiche, cit., p. XLV (" une mission "éclairée" que l'auteur perçoit comme l'élément fondamental et comme le devoir principal de l'intellectuel désormais libéré de l'étau de la culture médiévale").

71. Sin. nomine II, 3 : Quant au reste de l'Italie, qui doute qu'elle puisse réaliser tout ce dont elle a été capable par le passé et que ce ne sont ni la sagesse, ni les forces, ni les ressources, ni le courage qui lui manquent, mais une volonté commune? " (éd. cit., p. 38-39).

72. Cf. Dante, Épître VI, in Euvres complètes, , Paris, La Pochothèque, 1996, p. 529-533. Le titre est déjà révélateur de la tonalité de l'épître : "Dante Alighieri, Florentin et exilé injustement, aux Florentins très scélérats qui vivent à l'abri de leurs murs ". Citons deux extraits, Epist. VI, 5 et 6 : " Et 
tion différente du rôle de l'intellectuel. Pétrarque tourne son regard sur le terrestre et n'envisage aucune dimension prophétique dans sa démarche. À l'universalisme de Dante s'oppose désormais l'humanisme de Pétrarque : de la transcendance à l'immanence, du message sacré au message laïque, Pétrarque exploite le culte de la Rome antique tout en posant un regard à la fois prudent et pragmatique sur son époque. Pour preuve, la présence, que l'on pourrait définir paradoxale, dans le De remediis I, 116, d'affirmations absolument contraires au contenu des lettres d'exhortations adressées à Charles IV. Ainsi, lorsque Spes attend l'arrivée du prince, Ratio lui répond:

Et secum simul motus rerum varios, mutationes urbium, noxias novitates, famem, pestem, bella, discordias : hec vel universa vel singula modernis cum principibus venire sunt solita. ${ }^{73}$

Pétrarque semblait donc adhérer aux réserves de Charles IV, qu'il contestait pourtant avec emphase dans sa correspondance ; mieux valait pour l'Italie qu'il ne quittât pas son Allemagne. C'est peut-être cette conviction qui a poussé Pétrarque à ne pas faire entrer Charles IV dans le De viris illustribus, au profit de François de Carrare. Mais il ne pouvait pas négliger le poids de la tradition et aurait voulu néanmoins inscrire Charles dans la lignée des Scipions. Sans oublier une motivation personnelle : Pétrarque préférait qu'on lui reprochât une fidélité téméraire plutôt qu'un silence coupable $^{74}$. On ne peut toutefois pas nier la modernité de l'humaniste, et en conséquence le décalage avec la conception de Dante. En 1353, Pétrarque s'installe à Milan auprès de Giovanni Visconti ; un choix mal accepté par ses amis ${ }^{75}$, en particulier par Boccace qui y voit une trahison. Pétrarque savait que le système des communes était révolu et que la situation politique de l'Italie exigeait la main ferme d'un seigneur, voire d'un tyran, puisque l'idée impériale était en train de faire naufrage. Par ailleurs, il voyait en la personne de Giovanni Visconti l'idéal du prince-mécène, et fut le premier à percevoir à travers l'image du prince et de sa cour, un état unitaire capable de se développer selon la res publica romaine.

vous ne remarquez pas, parce que vous êtes aveugles, la cupidité qui vous domine, qui vous flatte par un chuchotement empoisonné, qui vous tient par des menaces fallacieuses, qui vous soumet à la loi du péché, et qui vous empêche d'obéir aux très saintes lois qui sont faites à l'image de la justice naturelle "; " reconnaissez que le temps de vous repentir amèrement de vos effronteries irréfléchies est arrivé " (traduction de Roberto Barbone et Antonio Stäuble).

73. De remediis I, 116, 10 : «Et tu verras arriver par la même occasion toutes sortes de bouleversements, des révolutions dans les villes, des nouveautés inquiétantes, la famine, la peste, la division ; tous ces dommages, qu'ils surviennent à la fois ou un par un, suivent ordinairement l'avènement des souverains modernes " (éd. cit., p. 508-511).

74. Cf. Fam. XXIII, 15, 3.

75. Cf. Fam. XVII, 10. 
Si Charles IV a entendu les exhortations pétrarquiennes d'une oreille peut-être plus bienveillante que réellement attentive, l'intellectuel a su se créer un chemin dans la diplomatie politique et apporter sa collaboration auprès des puissants ${ }^{76}$. Réciproquement ces puissants avaient besoin de lui et de cette nouvelle figure de l'intellectuel qu'il incarnait : un homme à la fois à l'intérieur et à l'extérieur de la vie politique, un homme qui privilégie son indépendance et sa liberté de penser, sans négliger les rapports avec autrui. Pétrarque jouissait désormais d'une renommée internationale, et faire partie de la liste de ses correspondants se rapprochait du privilège ${ }^{77}$. Il reste toutefois difficile de parler de politique pétrarquienne. Même dans les Sine nomine, l'écriture reste très littéraire et emphatique, les citations issues des Saintes Écritures et de la littérature antique sont fréquentes. Selon Ugo Dotti, certaines figures des Sine nomine, comme Nemrod, Julien l'Apostat, Cambyse ou Néron, ont aussi la fonction d'atténuer le contenu historico-politique de la dénonciation ${ }^{78}$. Pétrarque ne cherche pas à créer une polémique avec tout ce qu'elle pourrait entraîner en débats et en violences, il veut simplement faire entendre sa voix, participer aux affaires de la péninsule, sans pour autant s'encombrer de fonctions qui nuiraient à sa tranquillité. Par son rôle de médiateur, il peut concilier activités politiques, réflexions intellectuelles, dialogues avec les Anciens.

Dans les exhortations des Lettres familières, Pétrarque s'est directement adressé à celui qui détenait le pouvoir. Dans les Sine nomine en revanche, le destinataire fait partie du cercle d'amis proches de Pétrarque, et à ce propos Ugo Dotti parle d'un public « ormai disposto ad aprirsi a quelle nuove prospettive civili " ${ }^{79}$. Mais il ne s'agit pas de n'importe quel public. Pétrarque vise des gens cultivés, capables de comprendre et d'apprécier ses idéaux pour partager avec lui cette nouvelle culture qui s'éveille. Notre étude nous a ainsi permis de mettre en évidence que ce patrimoine humaniste a joué un rôle dans la persuasion politique; l'homme de lettres a su utiliser les exempla de l'Antiquité pour tenter de rétablir la grandeur de Rome, cultivant son éloquence et son érudition afin d'exhorter les puissants, et d'esquisser la nouvelle image de l'intellectuel.

76. Pétrarque va également devenir le médiateur dans la guerre fratricide et dévastatrice entre les républiques de Gênes et Venise. Cf. Fam. XI, 8 et XVIII, 16 au doge Andrea Dandolo ; Fam. XIV, 5 et 6 aux Génois ; Fam. XVII, 3 à Guido Sette, archevêque de Gênes sur la défaite des Génois, et XVII, 6 à Bernardo Anguissola, où il explique que Giovanni Visconti l'envoie pour régler le conflit.

77. Cf. la Fam. XIX, 2, où après avoir informé Zanobi da Firenze de son voyage auprès de Charles IV, Pétrarque précise que ses lettres sont interceptées par des voleurs, ce qui ne semble guère lui causer de soucis.

78. Sine nomine. Lettere polemiche e politiche, cit., p. XXXVIII.

79. Ibid., p. XLIV (un public « désormais disposé à s'ouvrir à ces nouvelles perspectives civiles »). 